\title{
Did Beta Canis Majoris Quit Pulsating?
}

\author{
Andrew P. Odell ${ }^{1,2,3}$, Robert D. Watson ${ }^{2}$ \\ ${ }^{1}$ University of Canterbury, Christchurch, New Zealand, \\ ${ }^{2}$ University of Tasmania, Hobart, Tasmania, Australia, \\ ${ }^{3}$ On sabbatical from Northern Arizona University
}

\begin{abstract}
The star $\beta$ CMa, prototype of the $\beta$ CMa variables, is known to have been a double mode pulsator since the beginning of the century, with amplitudes of 12 and $7 \mathrm{~km} / \mathrm{sec}$ for the 6.00 and 6.03 hour oscillations (Shobbrook, 1973). Recently, Dziembowski, Moskalik, and Pamyatnykh (1992) have produced models of $B$ stars using OPAL opacities which have many radial and non-radial modes unstable. As only a few modes are observed in these stars, these authors suggest that modes might change amplitude in such a way that modes can appear, disappear, or be replaced by other modes. Based on unsubstantiated rumour that $\beta \mathrm{CMa}$ had, like $\alpha \mathrm{Vir}$, quit pulsating (Pesnell, priv. comm.), we undertook observations with the Mt. John University Observatory (New Zealand) echelle spectrograph and CCD camera on the 1-m McLelland telescope. We found that $\beta \mathrm{CMa}$ is still pulsating, but with reduced amplitude and substantial period change compared with earlier data.

In particular, quadratic ephemerides fit to all known times of spectroscopic maxima agree with our times of maxima [P1: HJD 2448611.8436; P2: HJD 2448611.8278 ] to better than two minutes, but an earlier linear ephemeris by Chappelier (1985) disagrees by over two hours. Our data is best fit with amplitudes of about $7 \mathrm{~km} / \mathrm{sec}$ for both modes-this gives a nominal beat amplitude of nearly zero at the 49-day beat minimum (at a time when the linear ephemeris would have predicted a maximum amplitude). This may be the cause of the rumour mentioned above. Additional support for our conclusion of continuing pulsation comes from the fact that obvious line profile changes occur on the pulsation time scale.
\end{abstract}

\section{References:}

Chappelier, E., 1985, Astron. Astrophys. 147, 135.

Dziembowski, W. A., Moskalik, P., and Pamyatnykh, A. A., 1992, in proceedings of IAU Coll 137, Inside the Stars, Vienna, Austria, April 1992, in press.

Shobbrook, R. R., 1973, M.N.R.A.S. 161, 257. 